\title{
Uso de álcool e suicídio
}

\author{
Alcohol use and suicide \\ Raphael Eduardo Marques Gonçalves ${ }^{1}$, Júlio de Carvalho Ponce ${ }^{2}$, \\ Vilma Leyton ${ }^{3}$
}

\begin{abstract}
Gonçalves EMG; Ponce JC; Leyton V. Uso de álcool e suicídio. Saúde, Ética \& Justiça. 2015;20(1):9-14.
RESUMO: Suicídio refere-se a todas as causas de morte desencadeadas por uma ação da própria vítima contra si própria, com a ciência do resultado. É uma das principais causas de morte violenta em todo o mundo, e tornou-se uma questão de saúde pública, em virtude do aumento do número dos casos de suicídio entre os jovens, tanto nos países desenvolvidos quanto no Brasil. O consumo excessivo de álcool, a dependência alcoólica e o abuso de outras drogas estão intimamente ligados à tentativa ou consumação de suicídios. O objetivo deste estudo foi levantar o problema sobre o consumo de álcool por vítimas de suicídio no Brasil e no mundo, informação que é essencial para a formulação de políticas de prevenção à morte violenta relacionada ao consumo de álcool. A associação entre álcool e suicídio foi observada em estudos em vários países, inclusive no Brasil, com alcoolemias positivas, em média, em um terço das vítimas de suicídio. Apesar da considerável prevalência de suicídios no Brasil, faltam estudos que permitam uma abordagem epidemiológica para apoiar as políticas de saúde pública sobre esta questão. Da mesma forma, há pouca informação sobre o uso do álcool no Brasil e são escassos os estudos e os dados epidemiológicos que mostrem a associação entre os índices de mortalidade por causas externas e, em especial, por suicídio e uso do álcool, a fim de que sejam incluídos nas estratégias preventivas com o objetivo de reduzir o número de mortes violentas e os custos financeiros delas decorrentes.
\end{abstract}

DESCRITORES: Suicídio; Etanol; Toxicologia; Epidemiologia.

1. Perito Criminal da Polícia Técnico-Científica do Estado de São Paulo. Bacharel em Medicina.

2. Perito Criminal da Polícia Técnico-Científica do Estado de São Paulo. Bacharel em Ciências Moleculares. Mestre em Ciências pela Faculdade de Medicina da Universidade de São Paulo (FMUSP).

3. Professora Doutora do Departamento de Medicina Legal, Ética Médica e Medicina Social e do Trabalho da Faculdade de Medicina da Universidade de São Paulo (FMUSP).

Endereço para correspondência: Raphael Eduardo Marques Gonçalves. Rua Pamplona, 1112, apartamento 31, São

Paulo-SP, CEP: 01405-001. E-mail: raedu@yahoo.com 


\section{INTRODUÇÃO}

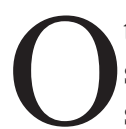

termo suicídio provém do latim sui, que significa "si próprio", e caedere, que significa "matar". Assim, suicídio referese a todas as causas de morte desencadeadas por uma ação da própria vítima contra si própria, com a ciência do resultado ${ }^{1}$.

O suicídio é uma das principais causas de morte violenta em todo o mundo e tem associação com fatores de risco socioeconômicos, médicos, culturais e psicossociais $^{2}$. Em 2000, a taxa mundial para mortes por suicídio foi 14,5/100.000 pessoas ${ }^{3}$. Recentemente, tem-se observado um aumento no número dos casos de suicídio entre os jovens, especialmente nos países desenvolvidos ${ }^{4}$. As taxas de suicídio no Brasil cresceram 21\% entre 1980 e 2000 para os homens na faixa etária de 15 a 24 anos de idade $^{5}$.

O suicídio pode estar relacionado a vários fatores precipitantes, tais como: problemas domésticos, perda do emprego, dificuldades financeiras e legais, doenças crônicas (incluindo as psiquiátricas como depressão e alcoolismo $)^{6,7}$. Muito se tem estudado sobre a etiologia do suicídio e do comportamento suicida, bem como os métodos utilizados para cometer suicídio ${ }^{8,9}$. Isso exige uma abordagem multidisciplinar com profissionais de diversas áreas do conhecimento: psicologia, psiquiatria, farmacologia, toxicologia, fisiologia, sociologia, ciências forenses e medicina legal ${ }^{10,11}$.

A morte prematura decorrente de suicídio gera repercussões com consequências econômicas e sociais, sendo que a identificação de indivíduos em maior risco é um dos principais desafios para os profissionais de saúde. Vários fatores de ordem psicossocial levam um indivíduo a cometer suicídio e um dos catalisadores é o consumo excessivo de álcool e a intoxicação alcoólica aguda ${ }^{12}$.

O consumo excessivo de álcool e/ou abuso de outras drogas estão intimamente ligados à tentativa ou consumação de suicídios, de acordo com estudo que analisou casos de suicídio por enforcamento e intoxicação exógena, no qual foi detectada alcoolemia positiva em $30 \%$ dos suicídios por enforcamento (média de 1,39 g/L) e em 36\% dos suicídios por intoxicação exógena (média de $1,39 \mathrm{~g} / \mathrm{L})^{13}$.

A embriaguez prejudica o juízo crítico e o autocontrole e as pessoas tendem a perder a inibição, tornando-se mais impulsivas e irresponsáveis, podendo chegar a autolesão e desencadear o comportamento suicida nos indivíduos com predisposição para tal, sendo amplamente conhecido que indivíduos com histórico de abuso de substâncias psicoativas estão sobrerrepresentados nas estatísticas de mortalidade ${ }^{14,15,16}$.

Suicídio sob a influência de álcool está fortemente associado com a dependência alcoólica segundo estudo realizado na Polônia ${ }^{17}$. Dessa forma, durante o diagnóstico e o tratamento de indivíduos com dependência alcoólica, o risco de depressão e ideação ou comportamento suicida devem ser cuidadosamente considerados ${ }^{18}$.

A questão da saúde mental também deve ser considerada, já que foi observado que transtornos psiquiátricos são duas vezes mais frequentes em pessoas que consomem álcool em relação aos abstêmios ${ }^{19}$. O consumo excessivo de álcool desempenha um papel importante em todas as formas de morte violenta: homicídio, suicídio e acidente ${ }^{20,21}$.

Embora o suicídio seja muitas vezes considerado como uma questão primordialmente de saúde mental, relacionando-se com transtornos afetivos em particular, estudos têm mostrado consistentemente que o suicídio e o comportamento suicida também são fortemente relacionados a distúrbios de dependência química, reconhecidamente para o álcool, bem como para outras drogas ${ }^{13,17,22-30}$. Estimou-se que o risco de suicídio é em média 10 vezes maior entre as pessoas com dependência química $^{30}$, sendo observado, em um estudo, um histórico de tentativa de suicídio em $43 \%$ dos pacientes internados para tratamento de dependência alcoólica ${ }^{31} \mathrm{e}$, em outro estudo, observou-se que a ideação suicida aumentou mais de três vezes e o risco de tentativas de suicídio mais quatro vezes em mulheres jovens com dependência alcoólica $^{32}$.

\section{OBJETIVO}

O objetivo deste artigo foi estudar a correlação entre a dependência de álcool, seu consumo agudo e as mortes por autolesão, informação que é essencial para a formulação de políticas de prevenção à morte violenta relacionada ao consumo de álcool.

\section{MATERIAL E MÉTODO}

No presente trabalho, foi realizado levantamento bibliográfico utilizando os descritores "suicide" e "alcohol" na base de dados Pub Med entre os anos 1980 e 2013. Foram também levantadas publicações em revistas afins e livros.

\section{RESULTADOS E DISCUSSÃO}

O consumo excessivo de álcool, a dependência alcoólica e o abuso de outras drogas estão intimamente ligados à tentativa ou consumação de suicídios. A associação entre álcool e suicídio foi observada em estudos em vários países no mundo, bem como no Brasil, com alcoolemias positivas, em média, em um terço das vítimas de suicídio.

No Brasil, o suicídio é um importante fator de mortalidade, porém é tratado como uma questão invisível, 
embora ocorram anualmente, em média, 7.800 mortes por esta causa, que é uma cifra considerável ${ }^{33}$. O álcool é considerado um importante fator de risco para acidentes de trânsito, suicídios, homicídios e outras causas de lesões, sendo a droga mais consumida pela população, pois seu uso é lícito, o acesso é fácil e o preço é baixo ${ }^{34}$. Dentre 153 países, o Brasil ocupa o $63^{\circ}$ lugar do uso per capita de álcool na faixa etária de $15 \operatorname{anos}^{33}$.

A associação entre uso de álcool e o suicídio foi observada em 10 países (Estados Unidos, Canadá, Finlândia, Alemanha Oriental, Suécia, Suíça, Bélgica, Holanda, Noruega, e Tchecoslováquia), mas não foi observada tal associação em três países (Dinamarca, Luxemburgo e Nova Zelândia) ${ }^{35}$. A mais forte associação entre consumo de álcool e suicídio foi observada na Suécia, onde os estudantes que ingeriam bebidas alcoólicas eram 31\% mais propensos a cometer suicídio em relação aos abstêmios ${ }^{36,37}$. Na Alemanha, 17\% das tentativas de suicídio foram associadas ao uso de álcool e $32 \%$ das tentativas, exitosas ou não, de suicídio se deram após ingestão aguda de álcool ${ }^{38}$. Foi observado que o alcoolismo é mais prevalente em vítimas de suicídio jovens do sexo masculino em relação aos mais velhos, relacionando-se com $56 \%$ dos casos de suicídio na Finlândia e com $37 \%$ das vítimas adolescentes na Suécia ${ }^{19}$.

Em um estudo realizado na Suécia, dentre os suicídios ocorridos no período do estudo, $71 \%$ eram do sexo masculino e $29 \%$ do sexo feminino. Referente ao consumo de álcool, $34 \%$ de todas as vítimas de suicídio haviam consumido álcool antes da morte, sendo que $36 \%$ dos homens e $31 \%$ das mulheres tinham uma alcoolemia positiva na necropsia. Muitas dessas vítimas apresentaram alcoolemia elevada, indicando consumo de grande quantidade de álcool próximo à morte. A alcoolemia média entre as vítimas de suicídio foi de 1,32 $\pm 0,89$ $\mathrm{g} / \mathrm{L}$, e a alcoolemia máxima foi de $6,6 \mathrm{~g} / \mathrm{L}$. A média de idade nos casos de alcoolemias positivas foi de 47 anos, em comparação com 54 anos nos casos de alcoolemias negativas. A prevalência de consumo de álcool em vítimas de suicídio foi maior em intoxicações exógenas $(45 \%)$ e asfixia por gás $(51 \%)$ e menos prevalente em precipitações $(19 \%)$ e lesões mecânicas $(18 \%)^{12}$.

Segundo as estatísticas do Centro de Controle e Prevenção de Doenças (EUA), 24\% das vítimas de suicídio tinham alcoolemia superior a $0,8 \mathrm{~g} / \mathrm{L}^{39}$. A prevalência do uso de álcool entre vítimas de suicídio no Estado do Novo México, EUA, foi de 44,3\%, sendo que a alcoolemia média dessas vítimas foi de 1,98 $\pm 0,88 \mathrm{~g} /$ $\mathrm{L}^{40}$.

No Reino Unido, entre 1988 e 1995, 45\% das vítimas de suicídio apresentavam alcoolemia positiva, com maior incidência na faixa etária de 35 a 44 anos $^{16}$. $\mathrm{Na}$ Irlanda, entre 2001 e 2002, 55,5\% das vítimas de suicídio haviam consumido bebidas alcoólicas antes de cometer suicídio, com alcoolemia média de 1,6 g/L $\mathrm{L}^{41}$. Na Austrália, 41\% dos suicidas apresentavam alcoolemia positiva (mediana de 1,0 g/L) e, dentre estes, 44\% eram homens e $27 \%$ mulheres e observou-se que a prevalência de álcool depende do método suicida utilizado ${ }^{42}$. Na Turquia, foi observada alcoolemia positiva em $12 \%$ das vítimas de suicídio por enforcamento, e a alcoolemia máxima aferida foi de 4,21 g/L em um alcoólatra crônico ${ }^{4}$. Na Finlândia, 36\% das vítimas de suicídio apresentaram alcoolemia positiva, sendo observada alcoolemia positiva duas vezes mais frequentemente em homens que em mulheres ${ }^{44}$.

Segundo estudos anteriores, o etanol foi a substância psicoativa mais comumente encontrada em amostras de sangue de suicidas por enforcamento e intoxicações por outras substâncias, sendo observado que em $62 \%$ dos casos de intoxicações e $66 \%$ dos enforcamentos a alcoolemia excedia $1,0 \mathrm{~g} / \mathrm{L}$, sugerindo que as vítimas estavam embriagadas quando de sua morte ${ }^{12,45,46}$

No Brasil, em estudo realizado com 290 vítimas de suicídios na região metropolitana de São Paulo no ano de 1994, observou-se que 36,2\% apresentavam alcoolemia positiva, sendo a que maioria das vítimas era do sexo masculino $(77 \%)$ e $44 \%$ pertenciam à faixa etária de 20 a 39 anos de idade ${ }^{47}$.

Foram analisados 632 casos de suicídio, ocorridos no ano de 2005 no Estado de São Paulo, Brasil, em que foram realizadas dosagens de alcoolemia, representando $39,1 \%$ do total de casos de suicídios ocorridos no mesmo período. Observou-se predominância do sexo masculino $(76,4 \%)$ em relação ao feminino $(23,6 \%)$, com $68 \%$ dos casos de suicídio na faixa etária de 20 a 49 anos de idade. No geral, o método suicida que apresentou maior prevalência foi o enforcamento ( $39,8 \%$ dos casos), sendo que, entre os homens, os principais métodos suicidas utilizados foram o enforcamento $(34,3 \%)$ e o ferimento por arma de fogo $(14,9 \%)$, enquanto que entre as mulheres foram a intoxicação exógena $(28,2 \%)$ e o enforcamento $(23,3 \%)$. Foi encontrada alcoolemia positiva em $37,1 \%$ dos homens e $20,1 \%$ das mulheres, sendo que a alcoolemia média para homens foi de 1,52 $\mathrm{g} / \mathrm{L}$ e para mulheres, 1,38 $\mathrm{g} / \mathrm{L}$. Os suicídios por intoxicação exógena apresentaram a maior alcoolemia média entre as causas selecionadas, com $1,78 \mathrm{~g} / \mathrm{L}$, o que sugere uma ingestão concomitante do agente tóxico e de etanol ${ }^{48}$. Assim, esses valores estão de acordo com o que alguns autores afirmam: que as pessoas têm maior ideação e comportamentos suicidas quando consomem álcool ${ }^{49}$. Há ainda indicações de que o consumo de álcool seria capaz de transformar a ideação suicida em ação, de forma que o consumo imediatamente antes do ato pode ser o fator desencadeante do evento fatal ${ }^{50}$.

Outro estudo também realizado no Estado de São Paulo, Brasil, evidenciou que há uma estreita correlação 
entre a morte violenta e o consumo de álcool, uma vez que, das 12.925 vítimas de mortes violentas necropsiadas no ano de 2006, 36,9\% apresentaram alcoolemia positiva, com média de 1,8 $\pm 1,0 \mathrm{~g} / \mathrm{l}$. A média de idade das vítimas foi de $36,7 \pm 16,2$ anos. Dentre as vítimas do sexo masculino, 39\% apresentaram alcoolemia positiva (média 1,8 $\pm 1,0 \mathrm{~g} / \mathrm{l}$ ), enquanto $21 \%$ das mulheres apresentaram esta positividade (média 1,5 $\pm 1,0 \mathrm{~g} / \mathrm{l}$ ). Os suicídios representaram $5,1 \%$ das mortes violentas em geral, sendo que dentre o total de vítimas de suicídio, $32,1 \%$ apresentaram alcoolemia positiva, com média de $1,6 \pm 0,8 \mathrm{~g} / \mathrm{1}^{33}$

Um estudo analisou 184 casos de vítimas de suicídio por enforcamento no ano de 2007 no Estado de São Paulo, Brasil, representando $10,7 \%$ do total de casos de suicídio no mesmo período. Observou-se predominância de vítimas do sexo masculino (84,2\%), com faixa etária média de 38 anos para ambos os sexos combinados, sendo que $67,4 \%$ dos casos tinham idades entre 15 e 44 anos em ambos os sexos. Foi encontrada alcoolemia positiva em $37,5 \%$ das vítimas $(41,3 \%$ em homens e $17,2 \%$ em mulheres), demonstrando que elas estavam sob a influência da ingestão aguda de álcool quando cometeram suicídio. A alcoolemia média para todos os casos positivos foi de 1,80 $\pm 0,90 \mathrm{~g} / \mathrm{L}$, o que é consistente com o comportamento autodestrutivo que pode levar ao suicídio, especialmente entre os homens ${ }^{51}$.

Dessa forma, segundo estudos analisados, observa-se uma relação dose-resposta, na qual: quanto maior o consumo de álcool, maior a prevalência de comportamentos suicidas ${ }^{52}$, sendo que o alcoolismo crônico está bastante relacionado com o suicídio, considerando que alcoólatras têm de 60 a 120 vezes mais probabilidade de cometerem suicídio do que a população abstêmia $^{50}$. Por outro lado, observou-se também que a redução do consumo de álcool em alguns países estava associada à queda no número de suicídios, enquanto, em outros, o alto consumo de álcool associava-se a taxas altas de suicídio ${ }^{36,53}$.

\section{CONCLUSÃO}

Existe uma relação entre o uso de álcool e a vitimização por suicídios. Entretanto, ao contrário do que existe na literatura internacional, ainda são poucos os estudos brasileiros que permitam uma abordagem epidemiológica para apoiar estratégias preventivas com o objetivo de reduzir o número de mortes por suicídios e de outras causas externas e seus custos financeiros relacionados.

Gonçalves EMG; Ponce JC; Leyton V. Alcohol use and suicide. Saúde, Ética \& Justiça. 2015;20(1):9-14.

\begin{abstract}
Suicide refers to all causes of death triggered by the victims' own actions, with knowledge of the final result. It is one of the main causes of violent deaths worldwide, and has become a public health issue, due to an increase in suicide among the young, in developed countries and Brazil. The excessive consumption of alcohol, alcohol dependence and other drug abuse are closely linked to attempted or completed suicides. The objective of the present study was to discuss the issue of alcohol consumption by suicide victims in Brazil and worldwide, information that is essential to the development of violent death prevention policies, especially those regarding alcohol consumption. The link between alcohol and suicide has been observed in studies around the world, as well as in Brazil, with positive alcohol samples being found, on average, in a third of the victims. Although there is a considerable prevalence of suicides in Brazil, studies regarding an epidemiological approach to this issue are scarce. Likewise, there is little information on alcohol use in Brazil, as well as studies and epidemiological data that show the association of death by external causes and especially, suicide and alcohol. This prevents potential suicide victims from being included in public policies aiming to reduce the number of suicides as well as the costs stemming from them.
\end{abstract}

KEY WORDS: Suicide; Ethanol; Toxicology; Epidemiology.

\title{
REFERÊNCIAS
}

1. Durkheim E. O Suicídio. Lisboa: Editorial Presença; 1982.

2. Sharma BR, Gupta M, Sharma AK, Sharma S, Gupta N, Relhan N, et al. Suicides in northern India: comparison of trends and review of literature. J Forensic Leg Med. 2007;14(6):318-26. DOI: http://dx.doi.org/10.1016/j. jcfm.2006.08.009

3. Krug EG, Dahlberg LL, Mercy JA, Zwi AB, Lozano R, editors. World report on violence and health. Geneva: World Health Organization; 2002.

4. Biddle L, Brock A, Brookes ST, Gunnell D. Suicide rates in young men in England and Wales in the 21st century: time trend study. BMJ. 2008;336(7643):539-42. DOI: http:// dx.doi.org/10.1136/bmj.39475.603935.25

5. Mello-Santos C, Bertolote JM, Yuan-Wang P. Epidemiology of suicide in Brazil (1980 - 2000): characterization of age and gender rates of suicide. Rev Bras Psiquiatr. 2005;27(2):131-4. DOI: http://dx.doi.org/10.1590/S151644462005000200011

6. Klerman GL. Clinical epidemiology of suicide. J Clin Psychiatry. 1987;48(12):33-8. 
Gonçalves EMG; Ponce JC; Leyton V. Uso de álcool e suicídio.

7. Pirkola SP, Isometsä, ET, Heikkinen, ME, Lönnqvist, JK. Suicides of alcohol misusers and non-misusers in a nationwide population. Alcohol Alcohol. 2000;35(1):70-5. DOI: http://dx.doi.org/10.1093/alcalc/35.1.70

8. Hawton $\mathrm{K}$, van Heeringen $\mathrm{K}$, Suicide. Lancet. 2009;373(9672):1372-81. DOI: http://dx.doi.org/10.1016/ S0140-6736(09)60372-X

9. Maris RW. Suicide. Lancet. 2002;360(9329):319-26. DOI: http://dx.doi.org/10.1016/S0140-6736(02)09556-9

10. Bertolote JM, Fleischmann A, De Leo D, Wasserman D. Psychiatric diagnoses and suicide: revisiting the evidence. Crisis. 2004;25(4):147-55. DOI: http://dx.doi. org/10.1027/0227-5910.25.4.147

11. Jemal A, Ward E, Hao Y, Thun M. Trends in the leading causes of death in the United States, 1970-2002. JAMA. 2005;294(10):1255-9. DOI: http://dx.doi.org/10.1001/ jama.294.10.1255

12. Holmgren A, Jones AW. Demographics of suicide victims in Sweden in relation to their blood-alcohol concentration and the circumstances and manner of death. Forensic Sci Int. 2010;198(1-3):17-22. DOI: http://dx.doi.org/10.1016/j. forsciint.2009.12.015

13. Jones AW, Holmgren A, Ahlner J. Toxicology findings in suicides: Concentrations of ethanol and other drugs in femoral blood in victims of hanging and poisoning in relation to age and gender of the deceased. J Forensic Leg Med. 2013;20(7):842-7. DOI: http://dx.doi.org/10.1016/j. jflm.2013.06.027

14. Brady J. The association between alcohol misuse and suicidal behaviour. Alcohol Alcohol. 2006;41(5):473-8. DOI: http://dx.doi.org/10.1093/alcalc/ag1060

15. Klimkiewicz A, Ilgen MA, Bohnert AS, Jakubczyk A, Wojnar M, Brower KJ. Suicide attempts during heavy drinking episodes among individuals entering alcohol treatment in Warsaw, Poland. Alcohol Alcohol. 2012;47(5):571-6. DOI: http://dx.doi.org/10.1093/alcalc/ags069

16. Crombie IK, Pounder DJ, Dick PH. Who takes alcohol prior to suicide? J Clin Forensic Med. 1998;5(2):65-8. DOI: http://dx.doi.org/10.1016/S1353-1131(98)90055-4

17. Fudalej S, Ilgen M, Fudalej M, Wojnar M, Matsumoto H, Barry KL, Ploski R, Blow FC. Clinical and genetic risk factors for suicide under the influence of alcohol in a Polish sample. Alcohol Alcohol. 2009;44(5):437-42. DOI: http:// dx.doi.org/10.1093/alcalc/agp045

18. Manninen L, Poikolainen K, Vartiainen E, Laatikainen T. Heavy drinking occasions and depression. Alcohol Alcohol. 2006;41(3):293-9. DOI: http://dx.doi.org/10.1093/alcalc/ agh246

19. Berglund M, Öjehagen A. The influence of alcohol drinking and alcohol use disorders on psychiatric disorders and suicidal behavior. Alcohol Clin Exp Res. 1998;22(7):333S45S. DOI: http://dx.doi.org/10.1111/j.1530-0277.1998. tb04388.x

20. Sjogren H, Eriksson A, Ahlm K. Role of alcohol in unnatural deaths: a study of all deaths in Sweden. Alcohol Clin Exp Res. 2000;24(7):1050-6. DOI: http://dx.doi.org/10.1111/ j.1530-0277.2000.tb04649.x

21. Poikolainen K. Alcohol and mortality: a review. J Clin Epidemiol. 1995;48(4):455-65. DOI: http://dx.doi. org/10.1016/0895-4356(94)00174-O
22. Borges G, Angst J, Nock MK, Ruscio AM, Walters EE, Kessler RC. Risk factors for twelve-month suicide attempts in the National Comorbidity Replication (NCS-R). Psychol Med. 2006;36(2):1747-57. DOI: http://dx.doi.org/10.1017/ S0033291706008786

23. Kausch O, McCormick RA. Suicide prevalence in chemical dependency programs: Preliminary data from a national sample, and an examination of risk factors. J Subst Abuse Treat. 2002;22(2):97-102. DOI: http://dx.doi.org/10.1016/ S0740-5472(01)00214-8

24. Kõlves K, Värnik A, Tooding LM, Wasserman D. The role of alcohol in suicide: A case-control psychological autopsy study. Psychol Med. 2006;36(7):923-30. DOI: http://dx.doi. org/10.1017/S0033291706007707

25. Mann JJ, Apter A, Bertolote J, Beautrais A, Currier D, Haas A. et al. Suicide prevention strategies: A systematic review. JAMA. 2005;294(16):2064-74. DOI: http://dx.doi. org/10.1001/jama.294.16.2064

26. Preuss UW, Schuckit MA, Smith TL, Danko GP, Buckman K, Bierut L, et al. Comparison of 3190 alcohol-dependent individuals with and without suicide attempts. Alcohol Clin Exp Res. 2002;26(4):471-7. DOI: http://dx.doi.org/10.1111/ j.1530-0277.2002.tb02563.x

27. Ries RK, Yuodelis-Flores C, Comtois KA, Roy-Byrne PP, Russo JE. Substance-induced suicidal admissions to an acute psychiatric service: characteristics and outcomes. J Subst Abuse Treat. 2008;34(1):72-9. DOI: http://dx.doi. org/10.1016/j.jsat.2006.12.033

28. Rossow I, Amundsen A. Alcohol abuse and suicide: A 40year prospective study of Norwegian conscripts. Addiction. 1995;90(5):685-91. DOI: http://dx.doi.org/10.1046/j.13600443.1995.9056859.x

29. Rudd MD. Warning signs for suicide? Suicide Life Threat Behav. 2003;33(1):99-100. DOI: http://dx.doi.org/10.1521/ suli.33.1.99.22790

30. Wilcox HC, Conner KR, Caine ED. Association of alcohol and drug use disorders and completed suicide: An empirical review of cohort studies. Drug Alcohol Depend. 2004;76S:S11-S19. DOI: http://dx.doi.org/10.1016/j. drugalcdep.2004.08.003

31. Wojnar M, Ilgen MA, Czyz E, Strobbe S, Klimkiewicz A, Jakubczyk A, et al. Impulsive and non-impulsive suicide attempts in patients treated for alcohol dependence. J Affect Disord. 2009;115(1-2):131-9. DOI: http://dx.doi. org/10.1016/j.jad.2008.09.001

32. Sundin M, Spak F, Spak L, Sundh V, Waern M. Substance use/abuse and suicidal behavior in young adult women: A population-based study. Subst Use Misuse. 2011;46(13):1690-9. DOI: http://dx.doi.org/10.3109/1082 6084.2011.605414

33. Sinagawa DM, Godoy CD, Ponce JC, Andreuccetti G, Carvalho DG, Muñoz DR, Leyton V. Uso de álcool por vítimas de morte violenta no Estado de São Paulo. Saúde, Ética \& Justiça. 2008;13(2):65-71. DOI: http://dx.doi. org/10.11606/issn.2317-2770.v13i2p

34. Andreuccetti G, de Carvalho HB, Ponce JC, Carvalho DG, Kahn T, Muñoz DR, et al. Alcohol consumption in homicide victims in the city of São Paulo. Addiction. 2009;104(12):1998-2006. DOI: http://dx.doi.org/10.1111/ j.1360-0443.2009.02716.x 
35. Lester D. Alcohol availability, alcoholism, and suicide and homicide. Am J Drug Alcohol Abuse. 1995;21(1):147-50. DOI: http://dx.doi.org/10.3109/00952999509095235

36. Sher L. Alcohol consumption and suicide. Q J Med. 2006;99(1):57-61. DOI: http://dx.doi.org/10.1093/qjmed/ hci146

37. Norström T. Alcohol and suicide: a comparative analysis of France and Sweden. Addiction. 1995;90(11):1463-9. DOI: http://dx.doi.org/10.1046/j.13600443.1995.901114634.x

38. Boenisch S, Bramesfeld A, Mergl R, Havers I, Althaus D, Lehfeld $\mathrm{H}$, et al. The role of alcohol use disorder and alcohol consumption in suicide attempts: a secondary analysis of 1921 suicide attempts. Eur Psychiatry. 2010;25(7):414-20. DOI: http://dx.doi.org/10.1016/j.eurpsy.2009.11.007

39. Morbidity and Mortality Weekly Report (MMWR). Alcohol and suicide among racial/ethnic populations 17 States 2005-2006. MMWR Morb Mortal Wkly Rep. 2009;58(23):637-41

40. May PA, Van Winkle NW, Williams MB, McFeeley PJ, DeBruyn LM, Serna P. Alcohol and suicide death among American Indians of New Mexico: 1980-1998. Suicide Life Threat Behav. 2002;32(3):240-55.

41. Bedford D, O'Farrell A, Howell F. Blood alcohol levels in persons who died from accidents and suicide. Ir Med J. 2006;99(3):80-3.

42. Darke S, Duflou J, Torok M. Toxicology and circumstances of completed suicide by means other than overdose. J Forensic Sci. 2009;54(2):490-4. DOI: http://dx.doi. org/10.1111/j.1556-4029.2008.00967.x

43. Üzun I, Büyük Y, Gürpinar K. Suicidal hanging: fatalities in Istanbul. Retrospective analysis of 761 autopsy cases. J Forensic Leg Med 2007;14(7):406-9. DOI: http://dx.doi. org/10.1016/j.jflm.2007.01.002

44. Ohberg A, Vuori E, Ojanpera I, Lonngvist J. Alcohol and drugs in suicides, Br J Psychiatry. 1996;169(1):75-80.

45. Hayward L, Zubrick SR, Silburn S. Blood alcohol levels in suicide cases. J Epidemiol Community Health. 1992;46(3):256-60.

46. Ingoldsby $\mathrm{H}$, Callagy $\mathrm{G}$. Alcohol and unnatural deaths in the West of Ireland: a 5-year review. J Clin Pathol. 2010;63(10):900-3. DOI: http://dx.doi.org/10.1136/ jcp.2010.078741

47. Carlini-Cotrim, B, Gallina JR, Chasin AAM. Ocorrências de suicídios sob efeito de álcool: um estudo na região metropolitana de São Paulo. Rev ABP-APAL. 1998;20(4):146-9.

48. Ponce JC, Andreuccetti G, Jesus MGS, Leyton V, Muñoz DR. Álcool em vítimas de suicídio em São Paulo. Rev Psiquiatr Clín. 2008;35(S1):13-6. DOI: http://dx.doi. org/10.1590/S0101-60832008000700004

49. Hufford MR. Alcohol and suicidal behaviour. Clin Psychol Rev. 2001;21(5):797-811. DOI: http://dx.doi.org/10.1016/ S0272-7358(00)00070-2

50. Sher L. Alcoholism and suicidal behavior: a clinical overview. Acta Psychiatr Scand. 2006;113(1):13-22. DOI: http:// dx.doi.org/10.1111/j.1600-0447.2005.00643.x

51. Zerbini T, Ponce JC, Sinagawa DM, Cintra RB, Muñoz DR, Leyton V. Blood alcohol levels in suicide by hanging cases in the state of Sao Paulo, Brazil. J Forensic Leg Med. 2012;19(5):294-6. DOI: http://dx.doi.org/10.1016/j. jflm.2012.02.022

52. Borges G, Rosovsky H. Suicide attempts and alcohol consumption in an emergency room sample. J Stud Alcohol. 1996;57(5):543-8. DOI: http://dx.doi.org/10.15288/ jsa.1996.57.543

53. Nemtsov A. Suicides and alcohol consumption in Russia, 1965-1999. Drug Alcohol Depend. 2003;71(2):161-8. DOI: http://dx.doi.org/10.1016/S0376-8716(03)00094-2 